\title{
Obésité et lipides
}

Oléagineux, Corps Gras, Lipides. Volume 10, Numéro 2, Mars 2003, Obésité et lipides

Auteur(s) : Bernadette Delplanque, INSERM, Comité de rédaction d`OCL., .

\section{ARTICLE}

Auteur(s) : Bernadette Delplanque, INSERM,

Comité de rédaction d'OCL.

L'estimation minimale de la prévalence de l'obésité chez l'adulte est passée en France de 5 \% en 1997 à plus de $10 \%$ en 2000, même si elle reste très inférieure à celle des Etats-Unis (20\% et $25 \%$ chez les hommes et femmes respectivement). Le problème est encore plus crucial pour les enfants et les adolescents dont surpoids et obésité auraient été multipliés par 4 depuis 1960 (prévalence de l'obésité estimée à $16 \%$ ). La mondialisation des systèmes alimentaires fait que ce problème concerne aussi de plus en plus les pays en voie de développement.

L'impact économique de l'obésité tient aux complications de la maladie elle-même et à son retentissement social d'autant plus négatifs que l'âge de l'apparition de l'obésité est de plus en plus précoce.

Les complications de l'obésité sont liées à des désordres endocrino-métaboliques multiples : entre autres, insulino-résistance, hypertension, dyslipidémies, regroupés plus généralement sous les termes de "syndrome pluri-métabolique " ou "syndrome $X$ ", qui conduisent à l'apparition du diabète de type II et secondairement à l'apparition de complications cardio-vasculaires. L'enjeu est donc important.

Dans ce dossier ont été abordées par des spécialistes les données les plus récentes concernant à la fois les mécanismes fondamentaux de l'obésité (nouvelles molécules impliquées, aspects physiologiques, cellulaires et moléculaires, rôle du système nerveux sympathique) et de certaines de ses conséquences (insulino-résistance).

Sans conteste, les études de ces dernières années sur le tissu adipeux ont montré que celui-ci était passé du statut d'organe de stockage à celui d'organe endocrine impliqué dans l'homéostasie énergétique mais ayant des fonctions pléïotropes capitales pour la survie de l'individu. Le problème est que l'Homme a résisté à des millénaires d'alternance de disette et d'abondance parce qu'il est programmé à stocker dans le tissu adipeux, mais qu'il est certaine-ment moins adapté (et il en paie les conséquences) lorsque le mode de vie comporte un régime pléthorique associé à une faible activité physique.

II n'existe que peu de cas d'obésités monogéniques chez l'Homme. L'obésité résulte d'un déséquilibre entre un excès d'apports et d'un défaut relatif en dépenses, d'où le rôle des interactions entre les multiples gènes impliqués et l'environnement nutritionnel. A ce jour, s'il existe différentes pistes de gènes "candidats" qui peuvent intervenir dans la prise alimentaire, la dépense énergétique, le 
développement de l'adipocyte ou le métabolisme des lipides, on sait que l'obésité " courante " résulte d'interactions entre l'environnement (nutrition) et les facteurs polygéniques de prédisposition qui peuvent être mis en évidence par une alimentation pléthorique mais aussi par la qualité de la réponse aux régimes restrictifs hypocaloriques.

Quant à trouver un traitement de l'obésité, il est tentant de penser que le tissu adipeux pourrait devenir pour les prochaines années une nouvelle cible thérapeutique pour ces molécules ou récepteurs découverts récemment et qui gèrent l'entrée et/ou la sortie des acide gras et autres lipides, mais ceci reste délicat et même potentiellement dangereux compte tenu des multiples fonctions vitales maintenant attribuées au tissu adipeux.

S'il est important de ne pas stigmatiser les obèses, ni de les enfermer dans un "ghetto diététicopsychologique ", ni de médicaliser l'alimentation quotidienne (voir texte de JP Poulain), reste donc à favoriser la prévention dans la population générale et surtout les populations à risque (enfants, adolescents), par une définition de l'équilibre entre apports énergétiques et dépenses et donc,... réduction des excès alimentaires et promotion de l'activité physique... 